\title{
Implementation of Two Level Scheduler in Cloud Computing Environment
}

\author{
Bagul Dhanashri R. \\ UG student \\ SSVPS's BS Deore College of Engineering \\ Dhule424005, \\ India
}

\author{
Toris Divya N. \\ UG student \\ SSVPS's BS Deore College of Engineering \\ Dhule424005, \\ India
}

\begin{abstract}
Cloud computing focuses on delivery of reliable, fault-tolerant and scalable infrastructure for hosting Internet based application services. Scheduling in cloud computing is responsible for selection of best suitable resources for task execution. Efficient task scheduling method can fulfill user's requirements, QoS, and improves the resource utilization; this increases the overall performance of the cloud computing environment. In two level scheduling first scheduler deals with virtual machine to host allocation and second scheduler deals with task to virtual machine.
\end{abstract}

\section{General Terms}

Cloud Computing, Scheduling

\section{Keywords}

Cloud Computing, Task Scheduling, Backfilling, Virtual Machine

\section{INTRODUCTION}

Cloud computing delivers infrastructure, platform, and software as services. Cloud computing is a type of parallel and distributed system which consist of collection of inter connected and virtualized computers that are dynamically provisioned and represented as one or more computing resources based on service level agreements which are established between service provider and customers[2]. Cloud computing provides Infrastructure as a Service model, in which user obtains the virtual machines as resource and deploy task on it. Cloud makes this a suitable platform to execute tasks in minimum time.

In cloud computing there are four deployment models. Public clouds are owned and operated by third parties. A Public cloud is that they may be larger than an enterprises cloud. This is based on "pay-as-you-go" model. Popular public clouds are Amazon Elastic Cloud Compute, Google App Engine. Private clouds are built exclusively for a single enterprise. Popular private clouds are IBM Smart Cloud Foundation and Microsoft Private Cloud. Hybrid Clouds combine both public and private cloud models. The Hybrid cloud environment is capable of providing on-demand, externally provisioned scale. Community Cloud shares computing infrastructure in between all the organizations which are of same community. For example, all Government organizations within the state of Maharashtra [14].

Cloud contain large amount of data .So, scheduling mechanism plays vital role in cloud computing. In this paper the system analyses scheduling technique for planning various types of jobs in cloud environment. Scheduling includes data canters, host, virtual machine and processing order of job for every resource in cloud. Two level scheduler focuses on optimizing system throughput by maximize overall resource utilization and increase performance of application and this system is used to perform efficient job scheduling in cloud.

\subsection{BACKFILLING STRATEGIES}

Backfill is a scheduling optimization which allows a scheduler to make better use of available resources by running jobs out of order. Backfilling allows smaller jobsto move forward in the schedule as long as such movement does not cause any other scheduled jobs to be further delayed.There are two backfilling strategies EASY and Conservative backfilling. In EASY (Extensible Argonne scheduling System) backfilling, only the first queued job is given Earliest Start Time [1]. Other approach is Conservative Backfilling every queued job is given guaranteed start time, so that it has a bounded delay [1].

\section{RELATED WORK}

Cloud Computing Architecture includes three layers, infrastructure layer, platform layer and application layer [10]. The infrastructure layer is a set of virtual hardware resources and related management function. Infrastructure as a service are Amazon EC2, Amazon S3[11].The platform layer is a set of software resources, which can provide an environment for developing, running, managing and monitoring cloud application to. Platform as a Services are Google Application Engine, Microsoft's Salesforce.com [12] .The application layer is user oriented; it implements the interaction mechanism between user and service provider with the support of platform Layer. Users can submit tasks through the application layer. Software as a service includes a complete software which are offered on the cloud. Users on cloud access software application hosted by the cloud vendor on the basis of pay-per-use. Software as a service areGmail and Microsoft's Hotmail, Google docs [11].

S.Selvarani, G.S. Sadhasivam proposed Improved Cost-Based Task Scheduling Algorithm that is able to measures resource cost as well as computational performance also improves by considering the factors cost and performance[5].

Ali Bagherinia proposed Fuzzy Neural Network Algorithm To avoid overloading for the host hold more resources, Improve resource utilization by considering the factors Resource Utilization, Load Balance [7].

Eakta Kumari and Monika proposed Round Robin, Priority Scheduling Algorithm for Low Response time and high throughput by considering the factors Resource selection for task execution[6].

GAN Guo-ning, HUANG Ting-lei, GAO Shuai proposed Genetic Simulated Annealing Algorithm for tasks Scheduling by considering QOS Parameters, Cost[8]. 


\section{SYSTEM ARCHITECTURE}

The system architecture contains cloud user, virtual machines, first scheduler, second scheduler, tasks. First role of cloud user is to input number of virtual machines that user wants to create on host then first scheduler will schedule those VM on hosts. Another role is to input various type of tasks (long-term or short-term) .After that second scheduler will schedule those input tasks on proper VM.

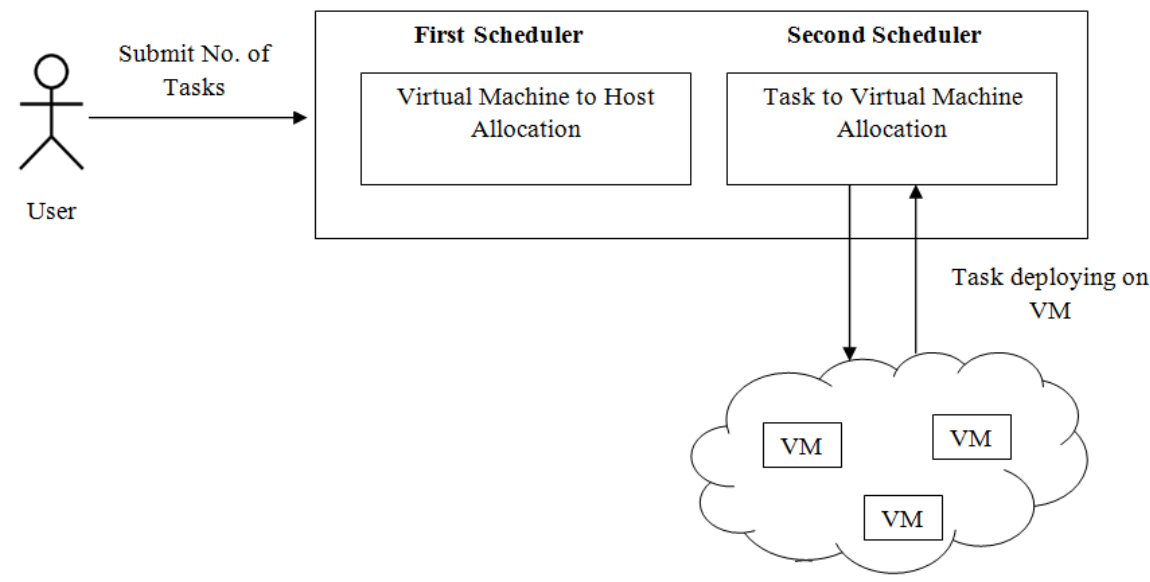

Fig 1: System Architecture

\section{SCHEDULING}

Scheduling is a biggest and challenging issue. The job scheduling algorithm is use to improve the performance and quality of service and minimize the total execution time. Job scheduling strategy must execute submitted number of jobs within a minimum time Job scheduling is an important task in cloud environment. Job Scheduling is used to allocate certain jobs to particular resources in minimum time. In cloud computing, job.

There are two types of scheduling. Static scheduling schedule tasks in known environment i.e. it already has the information about complete structure of tasks and mapping of resources before execution, estimates of task execution/running time. Dynamic scheduling must depend on not only the submitted tasks to cloud environment but also the current states of system and computer machines to make scheduling decision [4].

\subsection{Two Level Scheduler}

There are two schedulers,

\section{A. Virtual Machine to Host \\ B. Task to Virtual Machine}

\subsubsection{First Scheduler (Virtual Machine to Host)}

There are following steps to allocate VM to proper host:

Step-1.Creation of data center and host.

Step-2. Creation of VM.

Step -3. Schedule low configuration VM then high configuration of VM on host.

\subsubsection{Second Scheduler (Task to Virtual Machine)}

There are two types of jobs, Long-Term job and Short-term job. Length of long-term job in million instructions is greater than 100000 and length of short-term job in million instructions is less than or equal to 100000 [1].

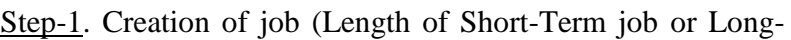
Term job).

$\underline{\text { Step-2 }}$. Scheduling of low and medium priority task on VM. High priority task goes into queue. Then high priority task will be scheduled on high configuration VM.

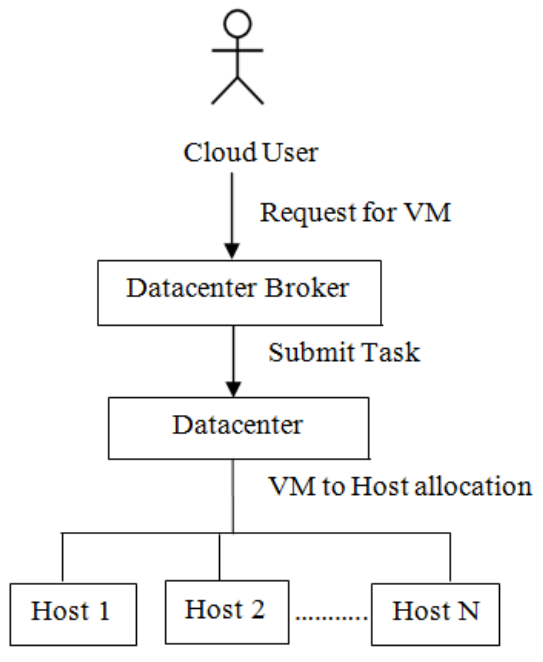

Fig 2: First Scheduler

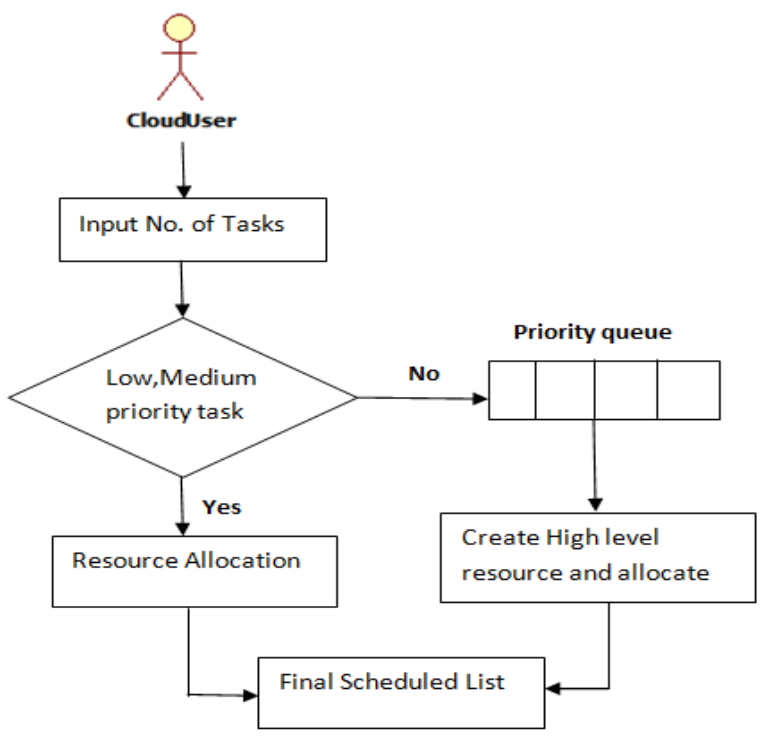

Fig 3: Second Scheduler 


\section{RESULT ANALYSIS}

\subsection{Cloudsim simulator}

CloudSim is a new open source toolkit developed using java including the predeveloped modules such as SimJava and GridSim. CloudSim offers the following features:

1. Modeling and simulating large scale data centers.

2. Modeling and simulating virtualized server hosts.

3. Modeling and simulating energy-aware computational resources.

4. A self-contained platform for modeling datacenters, datacenter brokers, scheduling and allocation policies [3].

5. Inserting simulation elements dynamically.

6. Availability of virtualization helps in creation and management of services [3].

7. Support for modelling and simulation of federated clouds.

CloudSim is a simulation tool for creating cloud computing environment and used as the simulator in solving the job scheduling problem. CloudSim allows us to create a data centre with a set of hosts and number of virtual machines as resources.

In this section we compare FCFS with two level scheduler. The below table 1 presents the comparison of scheduling algorithms FCFS and Two Level Scheduler

\begin{tabular}{|c|c|c|l|}
\hline Algorithm & Complexity & Allocation & $\begin{array}{c}\text { Waiting } \\
\text { Time }\end{array}$ \\
\hline $\begin{array}{c}\text { FCFS } \\
\text { Algorithm }\end{array}$ & $\begin{array}{c}\text { Simple } \\
\text { Scheduling } \\
\text { Algorithm }\end{array}$ & $\begin{array}{c}\text { CPU is } \\
\text { allocated in } \\
\text { the order in } \\
\text { which the } \\
\text { processes } \\
\text { arrive }\end{array}$ & $\begin{array}{l}\text { More for } \\
\text { Complex } \\
\text { job } \\
\text { submitted } \\
\text { last. }\end{array}$ \\
\hline $\begin{array}{c}\text { Two Level } \\
\text { Scheduler }\end{array}$ & $\begin{array}{c}\text { Complexity } \\
\text { depends on } \\
\text { the task to be } \\
\text { scheduled }\end{array}$ & $\begin{array}{l}\text { A. VM to } \\
\text { Host. } \\
\text { B.Task to } \\
\text { VM }\end{array}$ & Less \\
\hline
\end{tabular}

Table 1: Comparison of FCFS and Two Level Scheduler

\subsection{Simulation Description}

Virtual Machine - It is implemented virtual software of a computer that executes application programs same as a physical machine.

Cloudlet - Cloudlet is input job or set of tasks to be executed in cloud environment. Cloudlet has its own unique Cloudlet id, and Cloudlet length.

The simulation result is shows that, the two level scheduler showing remarkable output for number of input jobs as it is compare with FCFS with some changes. The algorithm of two level scheduler improves the performance of cloud system as implemented is simulation. Figure 4 and 5.Showing execution time of two level scheduler and FCFS implemented in this project in bar chart format.

Table 2 shows the execution time required by Two level scheduler and FCFS for short-term jobs.

\begin{tabular}{|c|c|c|c|}
\hline $\begin{array}{c}\text { No. Of } \\
\text { Tasks }\end{array}$ & $\mathbf{5}$ & $\mathbf{1 0}$ & $\mathbf{1 5}$ \\
\hline $\begin{array}{c}\text { Two Level } \\
\text { Scheduler }\end{array}$ & 33.19 & 66.88 & 415.33 \\
\hline FCFS & 58.61 & 94.25 & 442.70 \\
\hline
\end{tabular}

Table 2: Total Execution time for short-term jobs

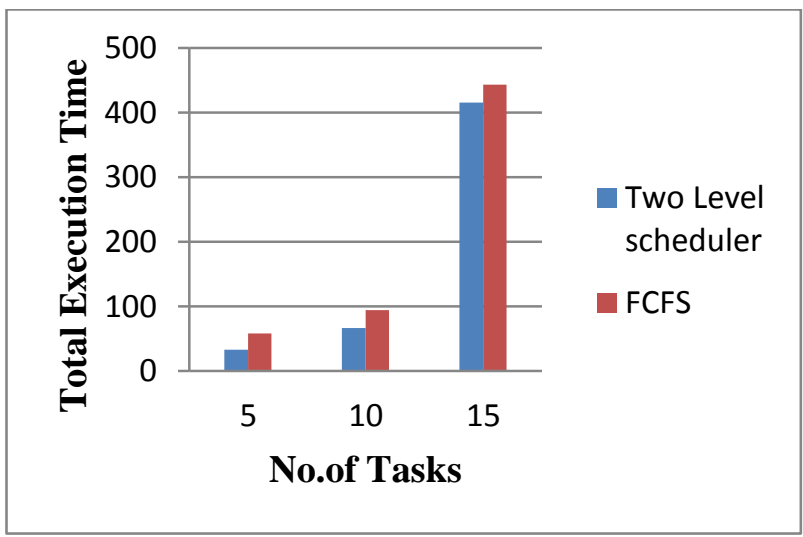

Figure 4: Total Execution time for FCFS and Two Level Scheduler (Short-Term job)

Table 3 shows the execution time required by Two level scheduler and FCFS for long-term jobs.

\begin{tabular}{|c|c|c|c|}
\hline $\begin{array}{c}\text { No. Of } \\
\text { Tasks }\end{array}$ & $\mathbf{5}$ & $\mathbf{1 0}$ & $\mathbf{1 5}$ \\
\hline $\begin{array}{c}\text { Two Level } \\
\text { Scheduler }\end{array}$ & 9577.90 & 16953.34 & 15728.11 \\
\hline FCFS & 9603.32 & 16980.71 & 15755.48 \\
\hline
\end{tabular}

Table 3: Total Execution time for Long-term jobs

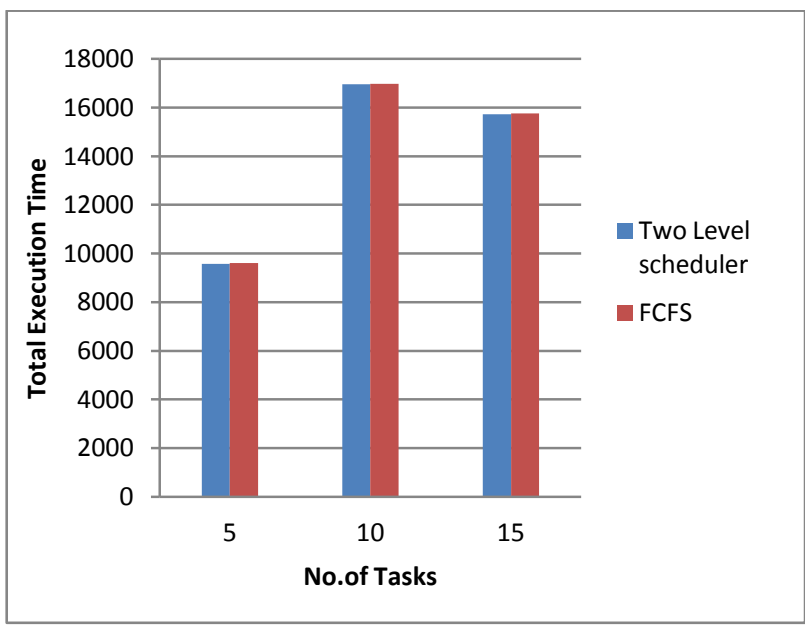

Figure 5: Total Execution time for FCFS and Two Level Scheduler (Long-Term job) 


\section{CONCLUSION}

Scheduling improves the server and resource utilization also to increases the performance of the host. In two level scheduler VM to host and task to VM allocation is done. Two level scheduler schedules the short-term as well as long-term jobs within a minimum time in cloud environment. The scheduling is more beneficial as compare to other method or algorithm because it can be able to schedule the jobs in good manner. The prioritized task should get preempt for specific number of time .Once it exceeds its number of preemption that task will get forcefully scheduled on that particular virtual machine.

\section{REFERENCES}

[1] R. J. Dr. Sudha Sadhasivam, "Design and implementation of an efficient two-level scheduler for cloud computing environment," International Conference on Advances in Recent Technologies in Communication and Computing, 2009.

[2] R. Buyya, C. S. Yeoa, S. Venugopala , J. Broberga, and I. Brandicc, "Cloud computing and emerging IT platforms: Vision, hype, and reality for delivering computing as the 5th utility," Future Generation Computer Systems, vol. 25, no. 6, pp. 599-616, 2009.

[3] R. Vijaya Lakshmi , Mrs. Soma Pratibha ,"Efficiant Two Level Scheduling in Cloud Computing Environment," IJMIE, Volume3, Issue5, May 2013.

[4] Raja Manish Singh, Sanchita Paul, Abhishek Kumar, "Task Scheduling in Cloud Computing: Review," Vol. 5 (6) , 2014

[5] S. Selvarani , G. S. Sadhasivam, "Improved cost-based algorithm for task scheduling in cloud computing," computational intelligence and computing research, pp.15,2010 .

[6] Eakta Kumari and Monika," A REVIEW ON TASK SCHEDULING ALGORITHMS IN CLOUD COMPUTING," International Journal of Science,
Environment and Technology, Vol. 4, No 2, 2015, 433 439

[7] Ali Bagherinia ,"A Task Scheduling Algorithm in Cloud Computing," International Journal of Recent Research in Mathematics Computer Science and Information Technology Vol. 2, Issue 1, pp: (310-313), Month: April 2015 - September 2015.

[8] GAN Guo-ning , HUANG Ting-lei, GAO Shuai , "Genetic Simulated Annealing Algorithm for task scheduling based on Cloud Computing Environment," 978-1-4244-6837-9/10/\$26.00 2010IEEE

[9] Rajveer Kaur , Supriya Kinger ,'Analysis of Job Scheduling Algorithms in Cloud Computing, "International Journal of Computer Trends and Technology (IJCTT) - volume 9 number 7 - Mar 2014

[10] Michael Armbrust, Armando Fox, Rean Griffith Anthony D. Joseph , Randy H. Katz , Andrew Konwinski , Gunho Lee , David A. Patterson, Ariel Rabkin , Ion Stoica , MateiZaharia ," Above the Clouds: A Berkeley View of Cloud Computing. In: EECS Department," University of California, Berkeley (2009).

[11] K. Venkata , Subba Reddy , Dr. A. MoizQyser , and J.Srinivas , "Cloud Computing Basics," International Journal of Advanced Research in Computer and Communication Engineering, vol. 1, no. 5, pp. 343-347, July 2012

[12] Torry Harris , "Cloud Computing - An Overview," SDA, pp. 1-6.

[13] Dr. Amit Agarwal , Saloni Jain ,"Efficient Optimal Algorithm of Task Scheduling in Cloud Computing Environment," International Journal of Computer Trends and Technology (IJCTT) - volume 9 number 7- Mar 2014

[14] Kalpana Parsi , M. Laharika ,"A Comparative Study of Different Deployment Models in a Cloud," International Journal of Advanced Research in Computer Science and Software Engineering, Volume 3, Issue 5, May 2013 